\title{
Full-Wave Analysis of Discontinuities in Conductor-Backed Coplanar Waveguides Using the Method of Lines
}

\author{
Shyh-Jong Chung, Member, IEEE, and Tun-Ruey Chrang
}

\begin{abstract}
A three-dimensional analysis using the method of lines with nonequidistant discretization is described to investigate the discontinuities in shielded conductor-backed coplanar waveguides (CBCPW's). An extended approach concerning the treatment of the boundary conditions at the input and output ports is proposed in which the reflection and transmission coefficients can be directively obtained in a single calculation. The validity and convergence of the numerical results are checked and gaps with various shapes in CBCPW's are analyzed and compared. Finally, the frequency response of a simple step discontinuity is calculated as an application to unsymmetrical structure.
\end{abstract}

\section{INTRODUCTION}

C OPLANAR waveguides (CPW's) possess several advantages over the conventional microstrip lines for monolithic or hybrid MIC applications. First, the characteristic impedance is primarily determined by the ratio of slot width and the center conductor, so size reduction is possible without limit. Second, they are virtually nondispersive and the radiation caused by discontinuities is reduced greatly. For open-circuited microstrip and CPW with similar sizes, the radiation loss of the latter is 20 to $40 \mathrm{~dB}$ less than that of the former [1]. Third, active devices can be easily inserted to the lines without using the via-hole technique. This simplifies the fabrication process and eliminates the inductions caused by the via holes. Fourth, it is easy to make transitions from CPW to other transmission lines, which makes for more flexibility in the design of circuits [2].

Several authors have paid their attentions to the analyses of the dispersion and impedance characteristics of coplanar waveguides, e.g., [3]-[6], but only a few tackle the discontinuity problems [7]-[11]. Naghed and I. Wolff [7] calculated the equivalent capacitance of coplanar waveguide discontinuities using the static finite difference method. Simons and Ponchack [8] obtained the equivalent circuit models by experiments. Among the full-wave analyses, Kuo and Itoh [9] applied the mode-matching technique to characterize the step junctions of shielded coplanar waveguides. Jackson [10] used the integral equation technique, with electric current bases, to consider the mode conversion at the open end of a finite-width conductorback coplanar waveguide. By using the same technique, but

Manuscript received September 14, 1992; revised March 1, 1993. This work was supported by the National Science Council of the Republic of China under Grant NSC 82-0404-E-009-336.

The authors are with the Institute of Communication Engineering, National Chiao Tung University, Hsinchu, Taiwan, Republic of China.

IEEE Log Number 9211852 with the bases of equivalent magnetic currents in the slots, Drissi et al. [11] analyzed the equivalent resistance and reactance of a CPW short end.

In this paper, we develop a Fortran program to study the discontinuities in the shielded conductor-backed coplanar waveguides using the method lines [12]. This method is well suited for analyzing three-dimensional resonators and periodic structures [13], [14]. But for the calculation of scattering parameters, additional treatment must be handled in the boundary conditions associated with the input and output ports. Worm [15] tackled the microstrip discontinuities by introducing inhomogeneous boundary conditions to the input port and assuming a short or an open circuit at the output port to obtain a homogeneous boundary condition. In his approach, three calculations of matrix equations were needed to obtain the whole scattering parameters. Chen and Gao [16] as well as Wu et al. [17] considered the same discontinuity problem, using hybrid boundary conditions. In the first case the incident and excited waves were tackled individually and in the second case only the reflection coefficient $\left(S_{11}\right)$ was shown.

In the present analysis we use the inhomogeneous boundary conditions but treat the reflection and transmission coefficients as unknowns. Two more equations are searched and added to the matrix equation obtained by the conventional procedure of the method of lines. The reflection and transmission coefficient are directively obtained from a Gaussian elimination without backsubstitution.

\section{FORMULATION}

Fig. 1 shows a discontinuity structure in the transition of two shielded conductor-backed coplanar waveguides. The waveguides are assumed to be single-moded and extend uniformly to infinity in the $+z$ and $-z$ directions, respectively. Let a wave (odd mode) propagate toward the discontinuity from $z=-\infty$, some of the power is reflected back to $z=-\infty$, and the other transmitted to $z=\infty$, both carried by the single propagation modes of the waveguides. Near the transition region higher order modes are excited, but vanish at the places far from the discontinuity. By considering the symmetries in the $x$ direction of the structure and the incidence condition, one may put a perfect magnetic conductor (PMC) at the plane of $x=A$, and thus reduce the solving space to the half region of $x>A$. 


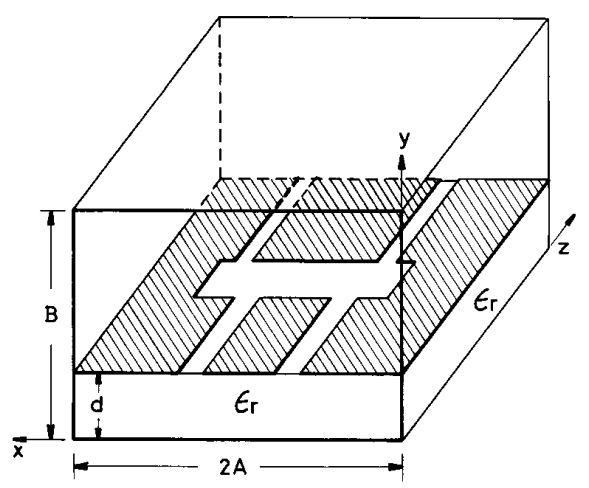

Fig. 1. Discontinuity in shielded conductor-backed coplanar waveguides.

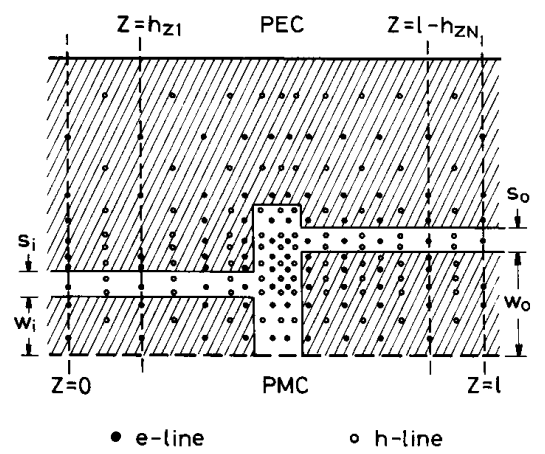

Fig. 2. Nonequidistant discretization in reduced solving region.

The two independent field components $E_{z}$ and $H_{z}$ in each separate layer shown in Fig. 1 should satisfy the Helmholtz equation

$$
\left(\frac{\partial^{2}}{\partial x^{2}}+\frac{\partial^{2}}{\partial y^{2}}+\frac{\partial^{2}}{\partial z^{2}}+k^{2}\right) \Psi=0
$$

where $\Psi$ stands for either $E_{z}$ or $H_{z}$, and $k^{2}=k_{o}^{2}$ (upper layer) or $\epsilon_{r} k_{o}^{2}$ (lower layer).

By the method of lines [12], the fields are discretized in the $x$ and $z$ directions and evaluated on the $e$-lines (for $E_{z}$ component) and $h$-lines (for $H_{z}$ component). Fig. 2 shows the locations of these lines in the reduced half space $(A x<$ $2 A, 0<z<l)$. It is noticed that the lines are made denser in the discontinuity region to consider the large variation of the field. At the input port $(z=0)$ and the output port $(z=l)$, the excited higher order modes are assumed to vanish so that only the dominant modes exist. This is also assumed to hold at the planes of $z=h_{z 1}$ and $z=l-h_{z N}$ shown in Fig. 2 .

The boundary conditions at the input port $(z=0)$ are

$$
\begin{aligned}
\left.E_{z}\right|_{z=0} & =(1-R) e_{z i} \\
\left.\frac{\partial H_{z}}{\partial z}\right|_{z=0} & =-j \beta_{i}(1-R) h_{z i}
\end{aligned}
$$

and at the output port $(z=l)$ are

$$
\begin{aligned}
\left.E_{z}\right|_{z=l} & =T e_{z o} \\
\left.\frac{\partial H_{z}}{\partial z}\right|_{z=l} & =-j \beta_{o} T h_{z o}
\end{aligned}
$$

where $e_{z i}\left(e_{z o}\right), h_{z i}\left(h_{z o}\right)$, and $\beta_{i}\left(\beta_{o}\right)$ are the modal fields and the propagation constant of the input (output) coplanar waveguide, which are calculated by the method of lines with one-dimensional discretization. $R$ and $T$ are the unknown voltage reflection coefficient and transmission coefficient, respectively.

Based on the boundary condition (2)-(5) and following procedures similar to [12] and [15], one may obtain the finite difference expression for the derivations of $E_{z}$ and $H_{z}$ with respect to $z$ as follows;

$$
\begin{aligned}
h_{z} \frac{\partial E_{z}}{\partial z} \rightarrow & \left(r_{z h} \overline{\boldsymbol{D}}_{z} \boldsymbol{r}_{z e}^{-1} \otimes \boldsymbol{I}_{x}\right) E_{z}+T\left(\boldsymbol{r}_{z h}^{2} \otimes \boldsymbol{I}_{x}\right) E_{z o} \\
& -(1-R)\left(\boldsymbol{r}_{z h}^{2} \otimes \boldsymbol{I}_{x}\right) \boldsymbol{E}_{z i} \\
h_{z} \frac{\partial H_{z}}{\partial z} \rightarrow & -\left(\boldsymbol{r}_{z e} \overline{\boldsymbol{D}}_{z} \boldsymbol{r}_{z h}^{-1} \otimes \boldsymbol{I}_{x}\right) \boldsymbol{H}_{z} \\
h_{x}^{2} \frac{\partial^{2} E_{z}}{\partial z^{2}} \rightarrow & -\hat{\boldsymbol{b}}_{e} \hat{\boldsymbol{P}}_{z e} \hat{\boldsymbol{b}}_{e}^{-1} \boldsymbol{E}_{z}-T\left(\boldsymbol{r}_{z e} \overline{\boldsymbol{D}}_{z}^{t} \boldsymbol{r}_{z h} \otimes \boldsymbol{I}_{x}\right) E_{z o} \\
& +(1-R)\left(\boldsymbol{r}_{z e} \overline{\boldsymbol{D}}_{z}^{t} \boldsymbol{r}_{z h} \otimes \boldsymbol{I}_{x}\right) \boldsymbol{E}_{z i} \\
h_{z}^{2} \frac{\partial^{2} H_{z}}{\partial z^{2}} \rightarrow & -\hat{\boldsymbol{b}}_{h} \hat{\boldsymbol{P}}_{z h} \hat{\boldsymbol{b}}_{h}^{-1} \boldsymbol{H}_{z}+T\left(\boldsymbol{r}_{z h}^{2} \otimes \boldsymbol{I}_{x}\right) \boldsymbol{H}_{z o}^{\prime} \\
& -(1-R)\left(\boldsymbol{r}_{z h}^{2} \otimes \boldsymbol{I}_{x}\right) \boldsymbol{H}_{z i}^{\prime}
\end{aligned}
$$

where the vectors $\boldsymbol{E}_{z}$ and $\boldsymbol{H}_{z}$ are the sampled values of $E_{z}$ and $H_{z}$ on the $e$-lines and $h$-lines, respectively, which are unknowns; $\otimes$ denotes the Kronecker products; $\hat{b}_{e}=r_{z e} \otimes r_{x e}$, $\hat{b}_{h}=\boldsymbol{r}_{z h} \otimes \boldsymbol{r}_{x h} ; \boldsymbol{r}_{z e}\left(\boldsymbol{r}_{x e}\right)$ and $\boldsymbol{r}_{z h}\left(\boldsymbol{r}_{x h}\right)$ are diagonal matrices associated with the nonequidistant discretization in the $z(x)$ direction, both turning into unit matrices for equidistant discretization [12] [15]; and

$$
\begin{aligned}
\boldsymbol{E}_{z i} & =\left[\begin{array}{l}
1 \\
\mathbf{0}
\end{array}\right] \otimes \boldsymbol{e}_{z i} \\
\boldsymbol{E}_{z o} & =\left[\begin{array}{l}
\mathbf{0} \\
1
\end{array}\right] \otimes \boldsymbol{e}_{z o} \\
\boldsymbol{H}_{z i}^{\prime} & =-j \beta_{i}\left[\begin{array}{l}
1 \\
\mathbf{0}
\end{array}\right] \otimes \boldsymbol{h}_{z i} \\
\boldsymbol{H}_{z o}^{\prime} & =-j \beta_{o}\left[\begin{array}{l}
\mathbf{0} \\
1
\end{array}\right] \otimes \boldsymbol{h}_{z o} \\
\hat{\boldsymbol{P}}_{z e} & =\overline{\boldsymbol{D}}_{z}^{t} \overline{\boldsymbol{D}}_{z} \otimes \boldsymbol{I}_{x} \\
\hat{\boldsymbol{P}}_{z h} & =\overline{\boldsymbol{D}}_{z} \bar{D}_{z}^{t} \otimes \boldsymbol{I}_{x} \\
\bar{D}_{z} & =\boldsymbol{r}_{z h} \boldsymbol{D}_{z} \boldsymbol{r}_{z e}
\end{aligned}
$$

with 0 being the zero vector and

$$
D_{z}=\left[\begin{array}{cccc}
1 & & & \\
-1 & \ddots & & \\
& \ddots & \ddots & \\
& & \ddots & 1 \\
& & & -1
\end{array}\right]
$$

The finite difference expressions for the derivatives of $E_{z}$ and $H_{z}$ with respect to $x$ are the same as those in [15]. By the transformations $\overline{\boldsymbol{E}}_{n z}=\hat{\boldsymbol{T}}_{e}^{t} \hat{\boldsymbol{b}}_{e}^{-1} \boldsymbol{E}_{z}$ and $\overline{\boldsymbol{H}}_{n z}=\hat{\boldsymbol{T}}_{h}^{t} \hat{\boldsymbol{b}}_{h}^{-1} \boldsymbol{H}_{z}$, one can transfer (1) to uncoupled differential equations: 


$$
\begin{aligned}
& \frac{\partial^{2}}{\partial y^{2}} \overline{\boldsymbol{E}}_{n z}-\left(\frac{\hat{\boldsymbol{\Lambda}}_{x e}^{2}}{h_{x}^{2}}+\frac{\hat{\boldsymbol{\Lambda}}_{z e}^{2}}{h_{z}^{2}}-k^{2}\right) \overline{\boldsymbol{E}}_{n z}=\frac{1}{h_{z}^{2}} \hat{\boldsymbol{T}}_{e}^{t} \\
& \cdot\left(\overline{\boldsymbol{D}}_{z}^{t} \boldsymbol{r}_{z h} \otimes \boldsymbol{r}_{x e}^{-1}\right)\left\{T \boldsymbol{E}_{z o}-(1-R) \boldsymbol{E}_{z i}\right\} \\
& \frac{\partial^{2}}{\partial y^{2}} \overline{\boldsymbol{H}}_{n z}-\left(\frac{\hat{\boldsymbol{\Lambda}}_{x h}^{2}}{h_{x}^{2}}+\frac{\hat{\boldsymbol{\Lambda}}_{z h}^{2}}{h_{z}^{2}}-k^{2}\right) \overline{\boldsymbol{H}}_{n z}=-\frac{1}{h_{z}^{2}} \hat{\boldsymbol{T}}_{h}^{t} \\
& \cdot\left(\boldsymbol{r}_{z h} \otimes \boldsymbol{r}_{x e}^{-1}\right)\left\{T \boldsymbol{H}_{z o}^{\prime}-(1-R) \boldsymbol{H}_{z i}^{\prime}\right\}
\end{aligned}
$$

where $\hat{T}_{h}$ and $\hat{T}_{e}$ denote the normalized eigenvector matrices of $\hat{\boldsymbol{P}}_{z h}$ and $\hat{\boldsymbol{P}}_{z e}$, and $\hat{\boldsymbol{\Lambda}}_{z h}^{2}$ and $\hat{\boldsymbol{\Lambda}}_{z e}^{2}$ denote their eigenvalue (quasi-) diagonal matrices, respectively. $\hat{\Lambda}_{x h}^{2}$ and $\hat{\Lambda}_{x e}^{2}$ are the counterparts of $\hat{\mathbf{\Lambda}}_{z h}^{2}$ and $\hat{\boldsymbol{\Lambda}}_{z e}^{2}$ in the $x$ direction.

By casting the solutions of (18) and (19) into the Maxwell's equations to obtain the other tangential field components, and employing the boundary condition at the interface of the two layers, one gets an inhomogeneous matrix equation in the transformed domain. This equation is then transformed back to the spatial domain to get

$$
\begin{aligned}
{\left[\begin{array}{l}
\boldsymbol{J}_{x} \\
\boldsymbol{J}_{z}
\end{array}\right]=} & \boldsymbol{Y}\left[\begin{array}{l}
\boldsymbol{E}_{x} \\
\boldsymbol{E}_{z}
\end{array}\right]-(1-R) \boldsymbol{Y}_{i}\left[\begin{array}{l}
\boldsymbol{E}_{x i} \\
\boldsymbol{E}_{z i}
\end{array}\right] \\
& -T \boldsymbol{Y}_{o}\left[\begin{array}{l}
\boldsymbol{E}_{x o} \\
\boldsymbol{E}_{z o}
\end{array}\right]
\end{aligned}
$$

After taking into account that the currents (tangential electric fields) vanish on the slot (conductor) region, a reduced matrix equation is obtained:

$$
\boldsymbol{Y}^{r}\left[\begin{array}{l}
\boldsymbol{E}_{x} \\
\boldsymbol{E}_{z}
\end{array}\right]_{s l o t}+R \boldsymbol{Y}_{i}^{r}\left[\begin{array}{l}
\boldsymbol{E}_{x i} \\
\boldsymbol{E}_{z i}
\end{array}\right]-T \boldsymbol{Y}_{o}^{r}\left[\begin{array}{l}
\boldsymbol{E}_{x o} \\
\boldsymbol{E}_{z o}
\end{array}\right]=\boldsymbol{Y}_{i}^{r}\left[\begin{array}{c}
\boldsymbol{E}_{x i} \\
\boldsymbol{E}_{z i}
\end{array}\right]
$$

If we assume that the numbers of $e$-lines and $h$-lines on the slot region are $N_{e}$ and $N_{h}$, respectively, then there will be $N_{e}+N_{h}$ linear equations in (21) but with $N_{e}+N_{h}+2$ unknowns. (Remember that $R$ and $T$ are treated as unknowns.) To obtain two additional equations we consider the currents at the planes of $z=h_{z 1}$ and $z=l-h_{z N}$. At these planes

$$
\begin{aligned}
\left.J_{z}\right|_{z=h_{z 1}} & =\left(e^{-j \beta_{i} h_{z 1}}-R e^{j \beta_{i} h_{z 1}}\right) j_{z i} \\
\left.J_{z}\right|_{z=l-h_{z N}} & =T e^{j \beta_{o} h_{z N}} j_{z o}
\end{aligned}
$$

where $j_{z i}$ and $j_{z o}$ are the $z$-directed modal currents of the input and output CPW's modes, respectively.

With (22) and (23), we may get two more sets of equations from (20): one corresponds to the currents at the plane of $z=h_{z 1}$, the other to those of $z=l-h_{z N}$. By averaging these two sets of equations individually, the required two equations are obtained. These equations together with (21) form a square matrix equations, which are then solved by the method of Gaussian elimination without backsubstitution to get the reflection coefficient $R$ and transmission coefficient $T$.

\section{NUMERICAL RESULTS}

In this section we present some numerical results derived form the above formulation. The structure parameters are chosen as $A=B=1.778 \mathrm{~mm}, d=0.254 \mathrm{~mm}, \epsilon_{r}=9.7$,

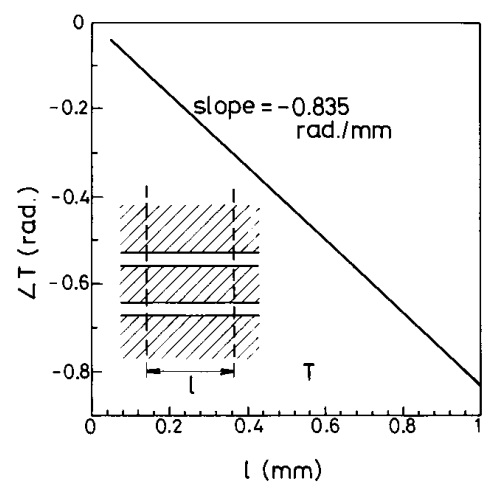

Fig. 3. Phase of the transmission coefficient as a function of the distance $(l)$ between input and output ports. $f=15 \mathrm{GHz}, \beta_{i}=\beta_{o}=0.835 \mathrm{rad} / \mathrm{mm}$.

$w_{i}=w_{o}=0.4 \mathrm{~mm}$, and $s_{i}=s_{o}=0.1 \mathrm{~mm}$. For the nonequidistant discretization, there are $N_{s} e$-lines and $N_{s}+1$ $h$-lines placed across the slot in the $x$ direction, and $N_{g} e$-lines and $N_{g}+1 j_{z i} h$-lines across the gap in the $z$ direction, both with a constant subinterval between any two adjacent $e$ - and $h$ lines. For lines outside the slot (in the $x$ direction) and the gap (in the $z$ direction), this subinterval increases as geometrical series with the quotient $q$ of successive subintervals being a constant. ( $q$ equals 1.2, except for the lines in the $x$ direction between the slot edge and the PMC, where $q$ equals 1.1.)

As a numerical check, Fig. 3 illustrates the variation of the phase delay from the input port to the output port for a through $\mathrm{CPW}$, as a function of the distance between the two ports. Although not shown here, the magnitude of the transmission coefficient $T$ is always calculated to 1 , and that of the reflection coefficient $R$ calculated to 0 (both exact to five digits). It is noticed that the results form a line with a slope equal to $0.835 \mathrm{rad} / \mathrm{mm}$, which is exactly the same as the propagation constant $\beta$ of the incident mode. This verifies the validity of the programming and the treatment of the source-based boundary conditions.

The convergence behavior of the reflection coefficient as a function of the interval size in the gap region is shown in Fig. 4. Here $P_{x}$ is fixed to 0.25 and $h_{x}=0.7 h_{z}$. It can be seen that both of the curves converge to the same value as the interval size decreases. The curve of $P_{z}=0.25$ enters the $0.5 \%$ precision region at $h_{z}=16 \mu \mathrm{m}$, where $N_{s}=9$ and $N_{g}=2$. In the following calculations, we set $P_{x}=P_{z}=0.25$, $N_{s}=10$, and the ratio of $h_{z} / h_{x}$ is appropriately chosen from 0.5 to 1.5 according to the gap width considered.

For a gap discontinuity shown in the inset, Fig. 5 displays the variations of the reflection coefficient $R$ and transmission coefficient $T$ as a function of the gap width $g$. When $g=0$, the gap vanishes, and all the power is transmitted to the output port $(|R|=0,|T|=1)$. As $g$ increases, the gap begins to reflect the power. The larger the gap is, the more power is reflected back. Only about $1 \%$ of the power is transmitted when the widths of the gap and slot become equal $(g=0.1 \mathrm{~mm})$. Notice that $h$ makes little influence on the reflection and transmission coefficients for the range (of $h$ ) considered. In all the calculations, $|R|^{2}+|T|^{2}$ are always equal 


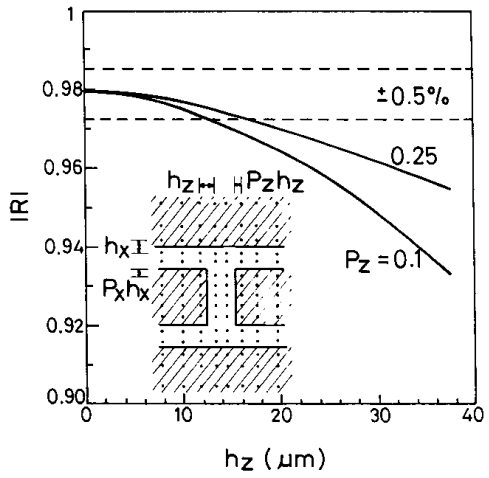

Fig. 4. Convergence behavior of the reflection coefficient as a function of the interval size in the gap region $\left(h_{z}\right)$ and the edge parameter $\left(P_{z}\right)$. Gap with $g=0.04 \mathrm{~mm} . P_{z}=0.25, h_{x}=0.7 h_{z} . f=15 \mathrm{GHz}$.

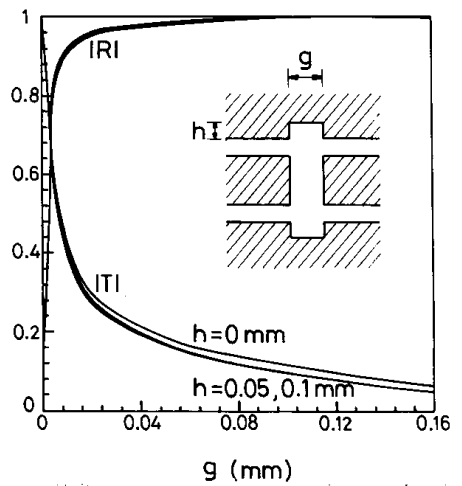

Fig. 5. Scattering parameters of gap discontinuities as a function of the gap width $(g) . f=15 \mathrm{GHz}$.

to 1 , which means that power is conserved. Actually, in our formulation the power conservation is satisfied no matter what the discontinuity is and how rough the discretization has been made.

Fig. 6 shows the frequency dependence of the reflection and transmission coefficients for the same structure as in Fig. 5. At low frequency, the quasi-static incident field sees an opened circuit at the gap, thus almost all the power is reffected back. But as the frequency increases, the wave begins to tunnel the gap, which makes more power transmitted to the output port.

The scattering characteristics of two closely spaced CPW step discontinuities are illustrated in Fig. 7. This configuration may be considered as a gap with a thin conductor connecting the center conductors of the input and output coplanar waveguides. Comparisons can be made between Figs. 7 and 5 . When the gap width $g$ approaches 0 , as expected, all the incident powers of both structures are transmitted to the output ports. But, due to the existence of the connecting conductor, the transmitted power in Fig. 7 remains high as $g$ increases, which is in contrast to those in Fig. 5. For the same structure, Fig. 8 displays the variations of the reflection and transmission coefficients as a function of the gap depth $h$. It is seen that, although increased with the gap depth, the reflection power is less than $1 \%$ of the incident power even when the width of

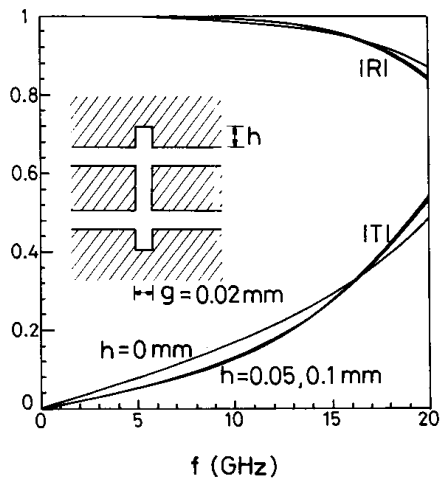

Fig. 6. Frequency dependence of the scattering parameters of gap discontinuities. $f=15 \mathrm{GHz}$.

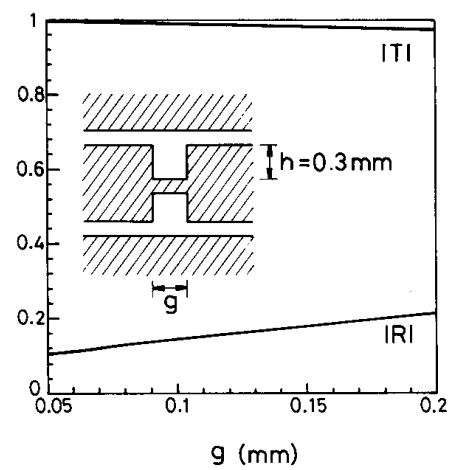

Fig. 7. Scattering parameters of two closely spaced step discontinuities as a function of the distance $(g)$ between the two steps. $f=15 \mathrm{GHz}$.

the connecting conductor shrinks to one fourth of that of the CPW's center conductor.

As an application to the unsymmetrical structure, Fig. 9 shows the frequency responses of the reflection and transmission coefficients of a step discontinuity. For each frequency considered, both the reflection and transmission coefficients are obtained in a single calculation.

\section{CONCLUSIONS}

The method of lines with two-dimensional nonequidistant discretization has been used to analyze the discontinuities in the conductor-backed coplanar waveguides. An extended approach concerning the treatment of the boundary conditions at the input and output ports is proposed. In this approach, the reflection and transmission coefficients are casted into the system equations as unknowns. Two additional equations have been found from the current distributions near the two ports. The scattering coefficients are obtained directively from a Gaussian elimination without backsubstitution. The validity of the method has been verified. Numerical results for CPW gaps with and without stubs and for two closely spaced CPW step discontinuities have been analyzed and compared.

In the programming of the formulation, a subroutine named "MASK" is developed to characterize the pattern of the metal (solt) in the metal plane. For tackling different discontinuity 


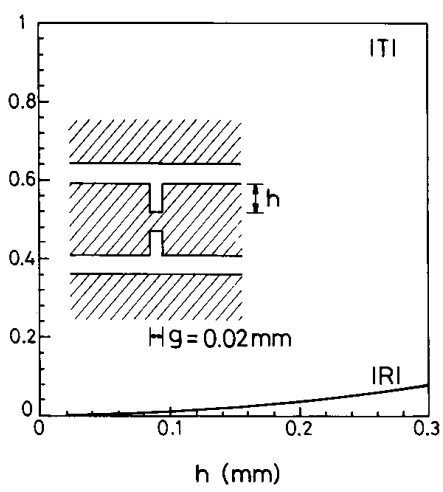

Fig. 8. Scattering parameters of two closely spaced step discontinuities as a function of the gap depth $h$ defined in the inset. $f=15 \mathrm{GHz}$.

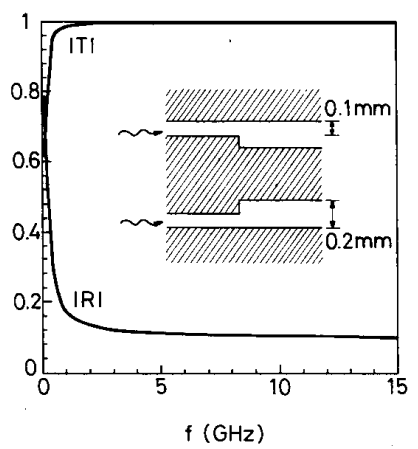

Fig. 9. Frequency dependences of the reflection and transmission coefficients of a step discontinuity.

problems, one only need to revise "MASK." This greatly reduces the analysis effort. By the present treatment of the boundary conditions, it is possible to deal with discontinuities in a multimoded shielded CPW, and this will be handled in the near future.

\section{REFERENCES}

[1] R.E. Stegens and D. N. Alliss, "Coplanar microwave integrated circuit for integrated subsystems," Microwave Syst. News and Commun. Tech. vol. 17, pp. 84-96, Oct. 1987.

[2] M. Riaziat, S. Bandy, and G. Zdasiuk, "Coplanar waveguides for MMIC's," Microwave J., p. 125, June 1987.

[3] Y.C. Shih and T. Itoh, "Analysis of conductor-backed coplanar waveguides," Electron. Lett., vol. 18, pp. 538-540, June 1982.

[4] M. Riaziat, R. Majidi-Ahy, and I-J. Feng, "Propagation modes and dispersion characteristics of coplanar waveguides," IEEE Trans. Microwave Theory Tech., vol. 38, pp. 245-250, Mar. 1990.

[5] D. A. Rowe and B. Y. Lao, "Numerical analysis of shielded coplanar waveguides," IEEE Trans. Microwave Theory Tech., vol. MTT-31, pp. 911-915, Nov. 1983.
[6] S.S. Bedair and I. Wolff, "Fast, accurate and simple approximate analytic formulas for calculating the parameters of supported coplanar waveguides for (M)MMIC's," IEEE Trans. Microwave Theory Tech., vol. 40, pp. 41-48, Jan. 1992.

[7] M. Naghed and I. Wolff, "Equivalent capacitances of coplanar waveguide discontinuities and interdigitated capacitors using a 3-dimensional finite difference method," IEEE Trans. Microwave Theory Tech., vol. 38, pp. 1808-1815, Dec. 1990.

[8] R. N. Simons and G.E. Ponchak, "Modeling of some coplanar waveguide discontinuities," IEEE Trans. Microwave Theory Tech., vol. 36, pp. 1796-1803, Dec. 1988.

[9] C.-W. Kuo and T. Itoh, "Characterization of shielded coplanar type transmission line junction discontinuities incorporating the finite metallization thickness effect," IEEE Trans. Microwave Theory Tech., vol. 40, pp. 73-80, Jan. 1992.

[10] R.W. Jackson, "Mode conversion at discontinuities in finite-width conductor-backed coplanar waveguide," IEEE Trans. Microwave Theory Tech., vol. 37, pp. 1582-1589, Oct. 1989.

[11] M. Drissi, V.F. Hanna, and J. Citerne, "Analysis of coplanar waveguide radiating end effects using the integral equation technique," IEEE Trans. Microwave Theory Tech., vol. 39, pp. 112-116, Jan. 1991.

[12] R. Pregla and W. Pascher, "The method of lines," in Numerical Techniques for Microwave and Millimeter Wave Passive Structures. T. Itoh, Ed. New York: Wiley, 1989, ch. 6.

[13] H. Diestel and S.B. Worm, "Analysis of hybrid field problems by the method of lines with nonequidistant discretization," IEEE Trans. Microwave Theory Tech., vol. MTT-32, pp. 633-638, June 1984.

[14] S. B. Worm and R. Pregla, "Hybrid-mode analysis of arbitrarily shaped planar microwave structures by the method of lines," IEEE Trans. Microwave Theory Tech., vol. MTT-32, pp. 191-196, Feb. 1984.

[15] S. B. Worm, "Full-wave analysis of discontinuities in planar waveguides by the method of lines using a source approach," IEEE Trans. Microwave Theory Tech., vol. MTT-38, pp. 1510-1514, Oct. 1990.

[16] Z. Chen and B. Gao, "Deterministic approach to full-wave analysis of discontinuities in MIC's using the method of lines," IEEE Trans. Microwave Theory Tech., vol. 37, pp. 606-611, Mar. 1989.

[17] K. Wu, M. Yu, and R. Vahldieck, "Rigorous analysis of 3-D planar circuit discontinuities using the space-spectral domain approach (SSDA)," IEEE Trans. Microwave Theory Tech., vol. 40, pp. 14751483, July 1992

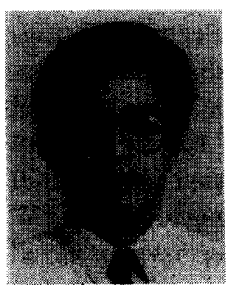

Shyh-Jong Chung (M'92) was born in Taipei, Taiwan, Republic of China, on January 18, 1962. He received the B.S.E.E. degree in 1984, and the Ph.D. degree in 1988, both from the National Taiwan University, Taipei, Taiwan.

Since 1988 he has been with the Department of Communication Engineering, National Chiao Tung University, Hsinchu, Taiwan, where he is currently an Associate Professor. His areas of interest include waveguide discontinuities, wave propagation, and numerical techniques in electromagnetics.

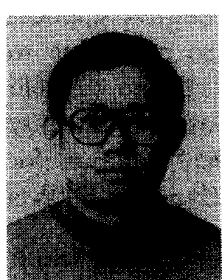

Tun-Ruey Chrang was born in Taiwan, Republic of China, on February 26, 1965. He received the B.S.E.E. degree from Christian Chung Yaun University, Chung Li, Taiwan, in 1988. Since September 1991 he has been working toward the M.S. degree at the Institutes of Communication, National Chiao Tung University, Hsinchu, Taiwan, R.O.C. His research work deals with electromagnetic theory and waveguide discontinuity problems. 